\title{
Rotor-to-Stator, Rub-related, Thermal/Mechanical Effects in Rotating Machinery
}

\author{
PAUL GOLDMAN and AGNES MUSZYNSKA
}

Bently Rotor Dynamics Research Corporation, P.O. Box 2529, Minden, Nevada 89423, USA

\begin{abstract}
The thermal effects of rotor-to-stator rub, and their influence on the rotor vibrational response, are discussed in this paper. Based on machinery observations, it is assumed in the analysis that velocities of transient thermal effects are considerably lower than that of rotor vibrations, and thermal effects affect only rotor steady-state vibrational responses. These responses would change due to thermally induced bow of the rotor, which can be considered slowly varying in time for the purposes of rotor vibration calculation. Thus uncoupled from the thermal problem, the rotor vibration is analyzed. The major consideration is given to the rotor, which experiences intermittent contact with the stator due to predetermined thermal bow of the rotor, unbalance force, and radial constant load force. In the case of an inelastic impact, this causes an on/off step-change in the stiffness of the system. A specially developed transformation is applied to the system model which contains discontinuities, and an averaging technique is then used to analyze stability of the different resonance regimes of rotor motion that were obtained. These regimes are further used to calculate the heat generated during rotor-to-stator contact stages, as a function of thermal conditions and rotor thermal bow modal parameters. The calculated heat input is used as a boundary condition for the rotor heat transfer problem. The latter is treated as quasi-static, which allows the application of an asymptotic method to the problem. The solution at its first approximation is used to adjust the rotor thermal bow value. As a result of this calculation, an ordinary differential equation with complex variables is obtained for the thermal bow, and it is investigated from the stability standpoint.
\end{abstract}

\section{NOTATION}

$a$

$A, B, G$

$b, u$

c

$C_{1}$

$C_{a}$

$D_{s}, M$

$D_{T}, \hat{K}, \hat{k}, \hat{h}$

E

$E_{1}, E_{2}, \hat{F}$

$f$

F

$g, \hat{g}$

h

$H, U$

$i, k$

I

$j=\sqrt{-1}$

$K_{1}, K_{2}, K_{3}$

$l, l_{1}, l_{2}$

$L$

$m, e, a$ nondimensional unbalance amplitude (17)

specific functions of the system parameters (33)

combined nondimensional unbalance amplitude and phase (25)

radial clearance between the rotor and stator

nondimensional amplitude of the rotor vertical response (22)

the root of resonance equation (31)

modal damping and modal mass of the rotor

thermal time constant and thermodynamic constants of the rotor

Young's modulus of elasticity

specific functions of the system parameters (48)

dry friction coefficient

generalized input (25)

heat rate density per unit area and its integral over the shaft length and cross section angle $\psi(19)$

nondimensional distance from the rotor to the stator

generalized input functions (17) and (25)

integer numbers

shaft moment of inertia

modal partial stiffnesses of the rotor

axial coordinate and its values for the disk and rub locations

length of the shaft

unbalance mass, radius and angular orientation 


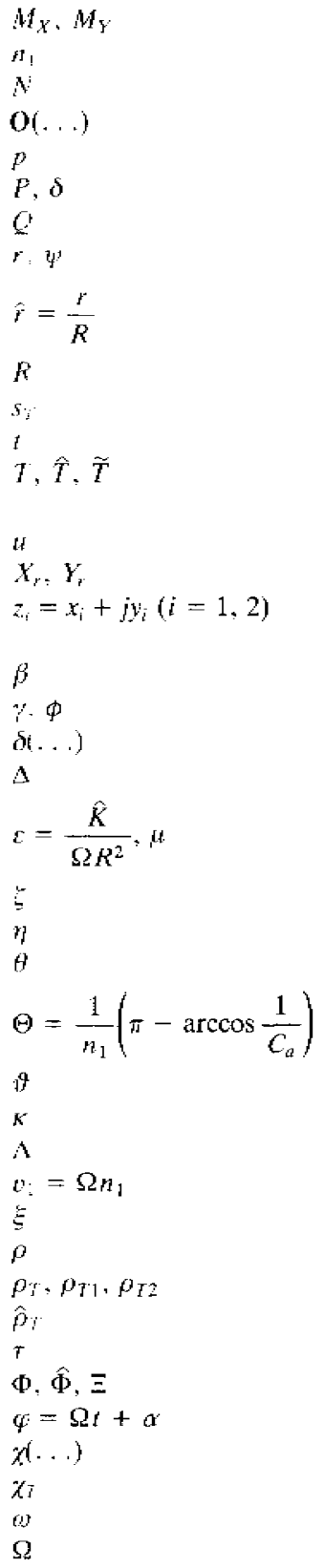

moments generated by normal thermal stresses

nondimensional natural frequency (17)

normal force at the rotor/stator contact

order of smallness

stiffness parameter of the system (17)

radial (side-load) force and its angular orientation respectively

generalized input (25)

polar coordinates of the shaft cross sections points

Nondimensional radial coordinate (19)

radius of the shaft cross section

nondimensional thermal bow (17)

time

temperature difference between specific point of the rotor and environment and its integrals ower the shaft length $l$ and cross-section polar coordinates $r$. $i$ (12) nondimensional horizontal displacement (17)

coordinates rotating with the rotor

rotor lateral displacements at the disk (1) and rub (2) locations in stationary

coordinates ( $x$-horizontal, $y$-vertical)

angular orientation of the thermal bow

slowly variable phases

Dirac function

nondimensional rotor/stator clearance coefficient

small parameters

second approximation to the vertical response nondimensionalized amplitude nondimensional damping (17)

phase of horizontal response

specific angle (36)

indicator of contact-no-contact between rotor and stator

modal coefficient (17)

special product of system coefficients (44)

lateral natural frequency of the rotor without rub (17)

detuning (30)

nondimensionalized amplitude of horizontal response

amplitude of thermal bow and its values at the disk and rub locations

vector of thermal bow in the coordinates rotating with the rotor

nondimensional time

phase of the rotor vertical response and its $2 \pi$-periodic functions (23)

disk rotating phase

unit step function

thermal expansion coefficient

frequency of the rotor vertical response to rotor rotative speed ratio

rotor rotative speed

\section{INTRODUCTION}

Rotor-to-stator rub, an unwelcome contact between a rotating and nonrotating elements of the machine, can be one of the most damaging malfunctions of rotating machinery. Generated by some perturbation of normal operating conditions that causes an increase of rotor vibration level, and/or an increase of the rotor centerline eccentricity, the rub can maintain itself, and gradually become more severe. The self-generating feature of this phenomenon originates from the interaction between rub-related thermal effects and lateral vibration response of the rotor. Starting from pioneering works of Tailor [1] and Newkirk [2], the unwinding spiral vibrations of rotors are documented in several papers [3-11]. In 
addition to that spiral response, Dimaragonas $[4,8]$ described an oscillating mode of shaft vibration, occurring during the transition from the spiraling to a steady state mode. $\Lambda$ similar result from an improved rotor dynamic model was obtained by Muszynska [11].

The problem of rub-related heat distribution was discussed by several authors, for example, Kellenberg [6], Smalley [10], Sweets [12]. The most complete analysis of the heat transfer problem associated with rub is given in the book by Dimaragonas and Paipetis [13]. In all referenced literature the analysis of the shaft bow, resulting from the uneven temperature distribution due to rub, is based on an approximation on the mean flexural rotation of one end of the shaft in relation to the other (Goodicr [14]).

The subject of this paper is an analysis of vibration responses of a rotor which experiences an occasional contact with the stator duc to the combination of thermal bow, radial load furce, and unbalance force. Machinery observations [11] indicate that the thermal processes are relatively slow; it is assumed, therefore, that they will aftect only the steady-state regimes of rotor vibration. This allows the time-dependent thermal problem to be uncoupled, and the analysis of the thermal problem and rotor vibration responses to be pursued independently.

The mathematical simulation of the pure vibrational aspect of rotor-to-stator rubs was considered in several papers [15-20]. In most cases of the mechanical/thermal problem, the analytical results are based on the method of harmonic balance, which is limited to periodic solutions, and is not quite strict mathematically. An attempt is made in this paper to adapt to the problem a more powerful asymptotic method, namely the averaging method. This method is valid only for the systems of ordinary differential equations in the specific forms [21]: the standard form and the form with multi-dimensional rotating phase. Mathematically, the problem reduces to establishing an appropriate transformation of the original variables in order to present the system in one of these forms. This problem has been solved using discontinuous variable transformation. The idea appeared first in the paper [22] by Zhuravlev, and was expanded later by Petchenev et al. [23] and Goldman et al. [24]. The developed mathematical body can further be applied to a number of other physical problems, including, for example, a problem of breathing and gaping shaft crack $[25]$.

\section{MATHEMATICAL MODEL OF THE RUBBING ROTOR}

An isotropic rotor at its first lateral mode is considered (Fig. 1). The rotor-to-stator rub occurs at the shaft axial location $l_{2}$. The mathematical model of the system is as follows:

$$
\begin{aligned}
M \ddot{z}_{1}+D_{\mathrm{s}} \dot{z}_{1}+\left(K_{1}+K_{2}\right)\left(z_{1}-\rho_{T 1} \mathrm{e}^{j(\varphi+\beta)}\right)-K_{2}\left(z_{2}-\rho_{T 2} \mathrm{e}^{j(\varphi+\beta)}\right) & =m \mathrm{e}^{2} \mathrm{e}^{j \varphi}-j P \\
K_{2}\left(z_{1}-\rho_{T 1} \mathrm{e}^{j(\varphi+\beta)}\right)-\left(K_{2}+K_{3}\right)\left(z_{2}-\rho_{T 2} \mathrm{e}^{j(\varphi+\beta)}\right)-\vartheta N(1+j f) \frac{z_{2}}{\left|z_{2}\right|} & =0, \\
j & =V-1, \cdot=\frac{\mathrm{d}}{\mathrm{d} t}
\end{aligned}
$$

where $K_{1}, K_{2}, K_{3}$ are modal partial stiffnesses of corresponding sections of the shatt (Fig. 1), $M, D_{\mathrm{s}}$ are modal mass and damping of the rotor, $\Omega$ is a rotor rotative speed, supposed constant; $z_{1}(t), z_{2}(t)$ are rotor complex lateral displacements at the disk and rub locations in stationary coordinates (see Notation); $\varphi=\Omega t+\alpha ; m, e, \alpha$ are unbalance mass, radius and angular orientation, respectively; $P$ is a constant radial (side-load) force, applied in the vertical-down direction; $f$ is a dry friction coefficient; $N$ is the normal force between the rotor and circular stationary obstacle, like a seal or stator. This force occurs during the rotor/stator contact stage. 


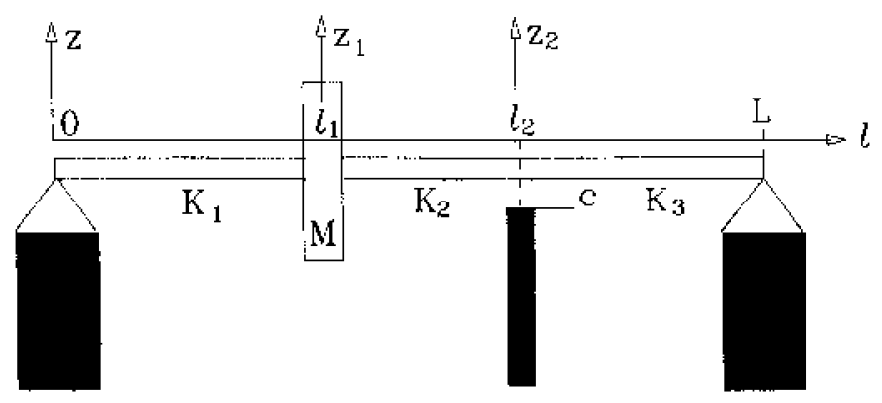

Fig. 1. Physical model of the rubbing rotor.

$$
\vartheta=\left\{\begin{array}{ll}
1 & \text { if }\left|z_{2}\right|=\sqrt{x_{2}{ }^{2}+y_{2}{ }^{2} \geqslant c} \\
0 & \text { if }\left|z_{2}\right|=\sqrt{x_{2}{ }^{2}+y_{2}{ }^{2}<c}
\end{array}\right\}
$$

is a rotor/stator contact indication function (a switch condition); $c$ is the radial clearance between the rotor and the stator at the rub location. The rub-related heating results in the thermal bow, distributed along the length of the shaft. It can be expressed in stationary coordinates (Fig. 1) as follows:

$$
\rho_{T}(l, t) \mathrm{e}^{j(t+\beta(t, t))}=\hat{\rho}_{T}(l, t) \mathrm{e}^{j \omega}
$$

where $l$ is an axial coordinate along the rotor (Fig. 1), $\beta$ is the thermal bow angular orientation, $\rho_{T}(l, t)$ is amplitude of bow at a particular axial location $l$ and time $t, \hat{\rho}_{T}(t, t)$ is the thermal bow vector in the coordinates rotating with the rotor (Fig. 2). As it will be shown later, in case of only one axial location of rub, the phase $\beta$ does not change with the

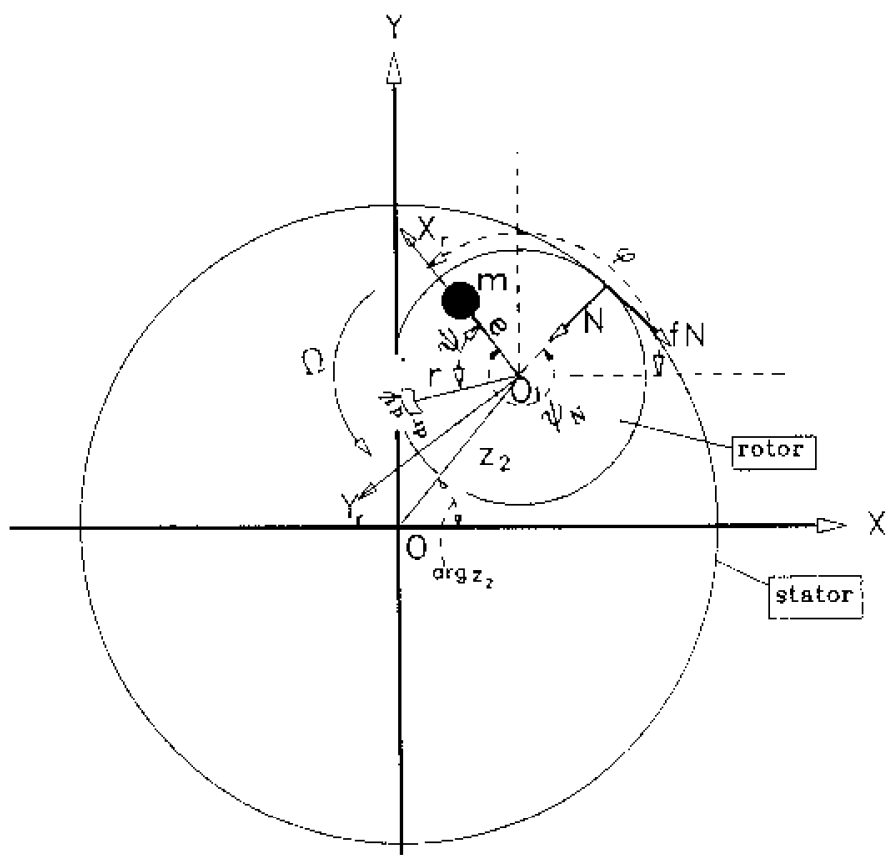

Fig. 2. Cross-section of the rotor at the rub axial location $l=l_{2}$. When $O O_{1} \equiv\left|z_{2}\right|=c$, the rotor-to-stator contact occurs, and normal force $N$ and tangential friction force $f N$ are applied to the shaft. $I X Y$ is inertial orthogonal system of coordinates with the shaft neutral axis as $/$ ( $O$ is a ptojection of this axis on this particular cross-section), $O_{1} X_{\mathrm{T}} Y_{\mathrm{T}}$ is an orthogonal system of rotating coordinates attached to the rotor; $r, \psi$ are polar coordinates. 
axial coordinate. For axial locations of the rotor disk and rubbing spot, the thermal bow differs only by a value of amplitude $\rho_{T i}=\rho_{T}\left(l_{i}, t\right)$. Note that the terms containing the thermal bow and unbalance force in Eqn (1) provide the energy for the rotor lateral vibration, while the terms describing damping and friction are responsible for the energy dissipation (the latter only during rotor-to-stator contact). The thermal bow appears due to an uneven temperature distribution along the rotor caused by the friction-force-generated heat. The latter can be characterized by the heat rate density $g(l, \psi, t)$ per unit area of the rotor cross section ( $\psi$ is an angular coordinate, Fig. 2). Considering the particular area element $R \mathrm{~d} l \psi$ ( $R$ is the rotor radius), on the rotor surface around the point with axial coordinate $l$, the friction force $f N$ is applied to the rotor if $\psi \leqslant \psi_{N} \leqslant \psi+\mathrm{d} \psi$ and $l \leqslant l_{2} \leqslant l+\mathrm{d} l$ (it is assumed that the rotor-to-stator contact occurs at one point only). The friction force, therefore, can be expressed in a form of a distribution over the rotor surface as follows:

$$
\vartheta f N=\vartheta f \int_{-L / 2}^{L / 2} \int_{0}^{2 \pi} \frac{N}{L} \delta\left(\frac{l}{L}-\frac{l_{2}}{L}\right) \delta\left(\psi-\psi_{N}\right) \mathrm{d} l \mathrm{~d} \psi
$$

where

$$
\psi_{N}=2 \pi-\left(\varphi-2 \pi\left[\frac{\varphi}{2 \pi}\right]\right)+\arg \left(z_{2}\right)
$$

describes the angular position of the friction force (Fig. 2), $[\varphi / 2 \pi]$ is the highest integer number $k$ which satisfies inequality $\varphi-2 \pi k \geqslant 0, \delta(\ldots)$ is the Dirac function. Since the rotor velocity at the contact location can be approximated as $R \Omega$, the heat rate density $g(l, \psi, t)$ per unit area equals to the friction force power per unit area. Taking into account Eqn (4), it can be presented as follows:

$$
g=f \Omega \frac{N}{L} \delta\left(\frac{l}{L}-\frac{l_{2}}{L}\right) \delta\left(\psi-\frac{3 \pi}{2}+\varphi-2 \pi\left[\frac{\varphi}{2 \pi}\right]\right) .
$$

\subsection{Thermal relations}

The thermal conductivity equation

$$
\frac{\partial^{2} T}{\partial r^{2}}+\frac{1}{r} \frac{\partial T}{\partial r}+\frac{1}{r^{2}} \frac{\partial^{2} T}{\partial \psi^{2}}+\frac{\partial^{2} T}{\partial l^{2}}=\frac{1}{\hat{K}} \frac{\partial T}{\partial t}
$$

with an initial condition

$$
\left.T\right|_{t=0}=0
$$

and boundary conditions

$$
\hat{k} \frac{\partial T}{\partial l}+\left.\hat{h} T\right|_{l=0, L}=0, \quad \hat{k} \frac{\partial T}{\partial r}+\left.\hat{h} T\right|_{r=R}=g(l, \psi, t)
$$

describes heating of the shaft due to rub. Here $T=T(r, \psi, l, t)$ is a difference of temperatures between the environment and the point of the shaft with coordinates $l, r, \psi$ at moment $t$, and $\hat{K}, \hat{k}, \hat{h}$ are thermodynamic constants. To complete the problem formulation, a relationship between the temperature distribution and thermal bow has to be derived. In order to accomplish this, assumptions are made that the rotor expansion in the axial direction is restricted, and that the rotor can be considered within the limits of Euler's beam theory. In this case, the normal thermal stress $\sigma_{l}$ in the axial direction can be expressed as follows:

$$
\sigma_{l}=\chi_{T} E T(l, r, \psi, t)
$$


where $\chi_{T}$ is the thermal expansion coefficient, $E$ is Young's modulus of elasticity. The moments related to the rotating coordinate axes $X_{r}, Y_{r}$ are as follows:

$$
M_{Y}=\chi_{T} \int_{0}^{2 \pi} \mathrm{d} \psi \int_{0}^{R} E T r^{2} \sin \psi \mathrm{d} r=M_{Y}(l), \quad M_{X}=\chi_{T} \int_{0}^{2 \pi} \mathrm{d} \psi \int_{0}^{R} E T r^{2} \cos \psi \mathrm{d} r=M_{X}(l) .
$$

For simplicity it is assumed that the shaft has constant modulus of elasticity $E$ and polar moments of inertia $I$ for all cross sections along its length. In order to find a displacement of the shaft at the axial location $l_{1}$, the theorem of Castigliano can be applied. For that purpose, a particular configuration of bearing supports was assumed (Fig. 3).

According to the Castigliano theorem and Eqns (10), the vector $\hat{\rho}_{T}(l, t)=\rho_{T}(l, t) \mathrm{e}^{j \beta l l t s}$ of the thermal bow in the rotating coordinates can be expressed as follows:

$$
\hat{\rho}_{T}(l, t)=\frac{\chi_{T} L}{I} \int_{0}^{L}\left(\frac{l+l_{a}-l-l_{a}}{2 L}-\frac{l_{a}}{L^{2}}\right)\left[\int_{0}^{2 \pi} \mathrm{d} \psi \int_{0}^{R} T \mathrm{e}^{j} r^{2} \mathrm{~d} r\right] \mathrm{d} l_{a}
$$

where $l_{a}$ is an integration variable. It is considered here a case of local heating when the temperature of the shaft differs from the environment only within a very narrow range of axial coordinates (Fig. 4).

Taking into account the rapid change of $T$ around $l_{2}$, the expression (11) can be estimated as follows:

$$
\hat{\rho}_{T}(l, t) \approx \frac{4 \chi_{T} L}{\pi R}\left(\frac{l+l_{2}-\left|l-l_{2}\right|}{2 L}-\frac{l_{2}}{L^{2}}\right) \tilde{T} ; \quad \tilde{T}=\int_{0}^{L}\left[\int_{0}^{2 \pi} \mathrm{d} \psi \int_{0}^{R} T \mathrm{e}^{\prime w^{\prime}} \frac{r^{2} \mathrm{~d} r}{R^{3}}\right] \mathrm{d} l .
$$

The expressions (12) have zero values at both ends of the rotor and a maximum at the point of heating $l=l_{2}$. Note that according to equations (12), the phase of the vector $\hat{\rho}_{T}$ does not depend on the axial coordinate.

Equations (1), (2), (5)-(8), and (12) fully describe the cross-coupled mechanical/thermal problem which is further analyzed in the next sections.

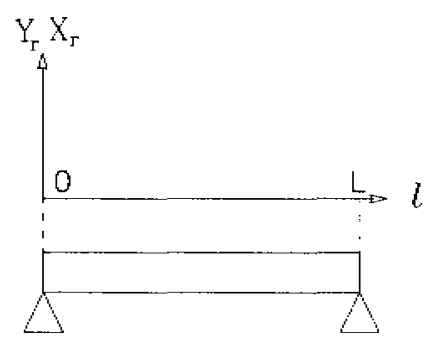

Fig. 3. System of coordinates and bearing supports.

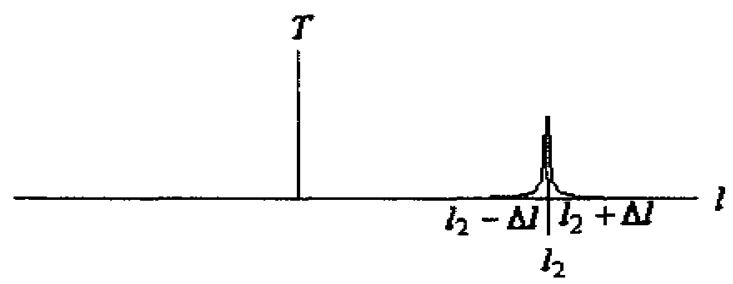

Fig. 4. Assumed temperature distribution for localized shaft heating. 


\subsection{Additional assumptions}

The following additional assumptions are made.

- Closeness of the rotor to the stator at the axial rub location is assumed. It means that the radial force can be expressed as follows:

$$
P=c\left[K_{1}\left(1+\frac{K_{3}}{K_{2}}\right)+K_{3}\right](1-\Delta), \quad \Delta \mid \ll 1, \quad \Delta \neq 0 .
$$

If the nondimensional clearance coefficient $\Delta$ is positive, then contact during static conditions does not occur: otherwise, the contact breaks only due to the dynamic action of the input forces.

- "Smaliness" assumptions: the static position of the rotor is perturbed by small forces, the thermal processes are slow:

$$
\Delta, f, \frac{D}{2 M \Omega}, \frac{\hat{K}}{\Omega R^{2}}=\boldsymbol{O}(\mu) ; \quad \frac{\rho_{T 2}}{c}, \frac{m e}{c\left(1+\frac{K_{3}}{K_{2}}\right) M}=\mathbf{O}\left(\mu^{2}\right)
$$

where $\mu$ is a small parameter.

\subsection{Transformation of the mathematical model}

An introduction of new variables $h=1 / \Delta\left(1-\sqrt{ }\left(x_{2}{ }^{2}+y_{2}{ }^{3}\right) / c^{2}\right)=\mathbf{O}(1)$ (rotor nondimetsional vertical displacement at rub location), and

$$
u=\frac{1}{c \Delta} \operatorname{Re}\left[\frac{z_{1}-\rho_{T 1} \mathrm{e}^{j(\varphi+\beta)}}{1+\frac{K_{3}}{K_{2}}}+\rho_{T 2} \mathrm{e}^{j(\varphi+\beta)}\right]=\mathbf{O}(1), \quad \text { Re }=\text { real part }
$$

( $u$ has a meaning of a rotor nondimensional horizontal displacement at the disk location) together with assumptions (13) and (14) allows equation (1) to be presented in the following form:

$$
\begin{aligned}
& h^{\prime \prime}+n_{1}{ }^{2}\left(1+\vartheta p^{2}\right) h-n_{1}{ }^{2}=\Delta H+\mathbf{O}\left(\mu^{2}\right), \quad u^{\prime \prime}+n_{5}^{2} u=\Delta U+\mathbf{O}\left(u^{2}\right), \\
& z_{2}=c\{-j+f+\Delta[u+j(1-\vartheta) h]\}+\mathbf{O}\left(\mu^{2}\right), \quad N=-j v_{c} \Delta\left(K_{2}+K_{3}\right)\left[h+\mathbf{O}\left(\mu^{2}\right)\right]
\end{aligned}
$$

where

$$
\begin{aligned}
& H=-\frac{a}{\Delta^{2}} \sin \varphi+\frac{s}{\Delta^{2}}\left(n_{1}{ }^{2}-\kappa\right) \sin (\varphi+\beta)-2 \frac{\eta}{\Delta} h^{\prime}-u^{\prime 2}+\frac{n_{1}{ }^{2} u^{2}}{2}=\mathbf{O}(1), \quad \frac{\mathrm{d}}{\mathrm{d} \tau}=\cdot \\
& U=\frac{a}{\Delta^{2}} \cos \varphi+\frac{s_{\mathrm{T}}}{\Delta^{2}}\left(n_{1}^{2}-k\right) \cos (\varphi+\beta)+y_{1}{ }^{2} p^{2} h\left(u+\frac{f}{\Delta}\right)-2 \frac{\eta}{\Delta} u^{\prime}=\mathbf{O}(1) \text {, } \\
& \tau=\Omega t_{1} \quad \frac{D_{s}}{2 M \Omega}=\eta=\mathbf{O}(\mu), \quad \frac{m e}{c\left(1+\frac{K_{3}}{K_{2}}\right) M}=a=\mathbf{O}\left(\mu^{2}\right), \quad \frac{\rho_{T 2}}{c}=s_{T}=\mathbf{O}\left(\mu^{2}\right),
\end{aligned}
$$

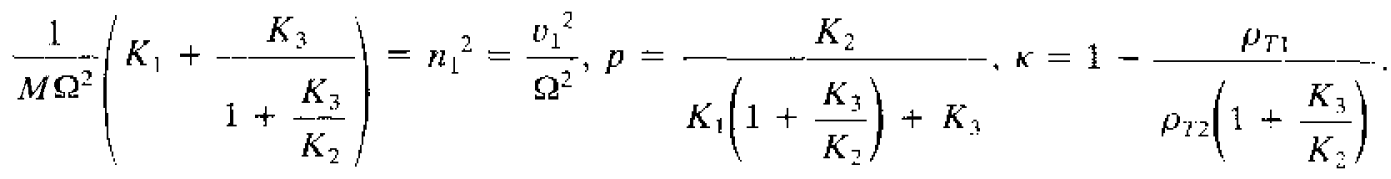


The conditions (2) of the switch become now very simple:

$$
\vartheta= \begin{cases}0 & \text { if } h>0 \\ 1 & \text { if } h \leqslant 0\end{cases}
$$

Note that the right-side terms of equations (16) are calculated with the accuracy up to the second order of smallness, but the conditions (18) of the switch are precise. Equations (16) are derived with the assumption (14) of the thermal process slowness. The latter can also be incorporated in equations (6), (8) as follows:

$$
\begin{gathered}
\frac{\partial \hat{T}}{\partial \tau}=\varepsilon\left(\frac{\partial^{2} \hat{T}}{\partial \hat{r}^{2}}+\frac{1}{r} \frac{\partial \hat{T}}{\partial \hat{r}}-\frac{\hat{T}}{\hat{r}^{2}}\right) \\
\frac{\hat{k}}{R} \frac{\partial \hat{T}}{\partial \hat{r}}+\left.\hat{h} \hat{T}\right|_{\hat{r}=1}=\hat{g}(t), \quad \hat{g}(t)=\int_{0}^{L} \mathrm{~d} l \int_{0}^{2 \pi} g \mathrm{e}^{j \psi} \mathrm{d} \psi=j f \Omega c\left(K_{2}+K_{3}\right) \Delta h \mathrm{e}^{-j \phi}
\end{gathered}
$$

where $r=R \hat{r}$,

$$
\varepsilon=\frac{\hat{K}}{\Omega R^{2}}=\mathbf{O}(\mu)
$$

and

$$
\hat{T}(\hat{r}, t)=\int_{0}^{2 \pi} \mathrm{d} \psi \int_{0}^{L} T \mathrm{e}^{j \psi} \mathrm{d} l .
$$

Since, according to equation (12), $\hat{\rho}_{T}$ is proportional to the function of temperature $\widetilde{T}=\int_{0}^{1} \hat{T} \hat{r}^{2} \mathrm{~d} \hat{r}$, it follows from the first equation (19) that $\mathrm{d} \hat{\rho}_{T} / \mathrm{d} t=\mathbf{O}\left(\mu \hat{\rho}_{T}\right)=\mathbf{O}\left(\mu^{3}\right) . \Lambda \mathrm{s}$ a consequence, equations (16) contain the thermal bow as a parameter, and the system mechanical model is thus essentially decoupled from the thermal part of the problem.

\section{PRESENTATION OF THE SYSTEM MATHEMATICAL MODEL IN THE FORM CONTAINING A MULTI-DIMENSIONAL ROTATING PHASE}

Since the right-side terms of equations (16) have higher order of smallness than the left-side ones, the generating system defined as equations (16) without right-side terms is as follows:

$$
\begin{aligned}
& h^{\prime \prime}+n_{1}^{2}\left(1+\vartheta p^{2}\right) h-n_{1}^{2}=0 \\
& u^{\prime \prime}+n_{1}^{2} u=0 .
\end{aligned}
$$

The second equation (20) has a very simple solution:

$$
u=\rho \cos \theta, \quad \rho^{\prime}=0, \quad \theta^{\prime}=n_{\mathrm{I}} .
$$

The first equation (20), together with the switch conditions (18), is more complex. Its solution can be built using piecewise integration, and connecting conditions at the ends of continuity intervals:

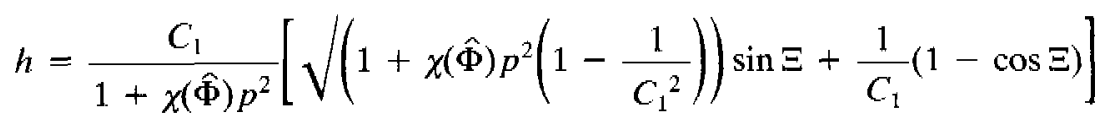


where

$$
\Phi=\omega\left(C_{1}\right) \tau+\alpha
$$

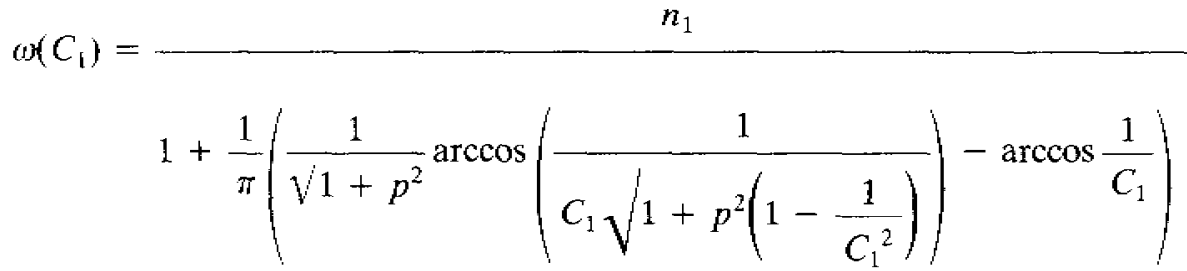

$$
\begin{aligned}
& \Xi=\frac{n_{1} \sqrt{1+\chi(\hat{\Phi}) p^{2}}}{\omega}\left\{\Phi-2 \pi\left(\left[\frac{\Phi}{2 \pi}\right]+\chi(\hat{\Phi})\right)\right\}, \\
& \hat{\Phi}=\Phi-2 \pi\left[\frac{\Phi}{2 \pi}\right]-2\left(\pi-\arccos \frac{1}{C_{1}}\right) \frac{\omega}{n_{1}} \text {. }
\end{aligned}
$$

The relation between $h$ and $C_{1}$ and $\Phi$ is shown graphically in Fig. 5 .

Equation (22) with the notation (23) constitutes the transformation of variables. This transformation produces the rotor vertical response 'amplitude' $C_{1}$ and 'phase' $\Phi$. Since the zero value of $\Phi$ is determined at the instant the rotor breaks the contact with the stator, $\Phi$ is related in a certain way to the rotating phase $\varphi$ which serves as a time reference.

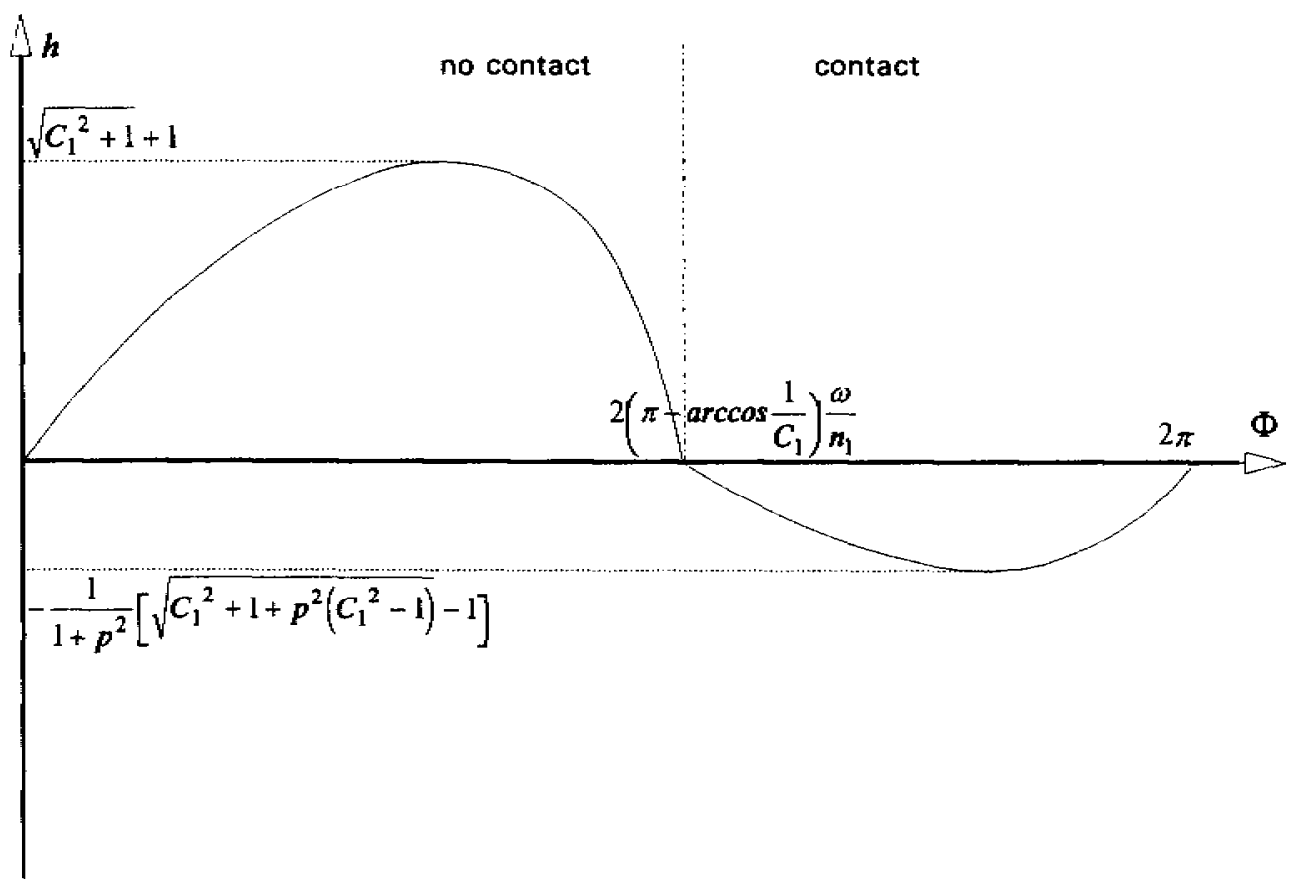

Fig. 5. The nondimensional distance between the rotor and stator

$$
h=\frac{1}{\Delta}\left(1-\sqrt{\frac{x_{2}^{2}+y_{2}^{2}}{c^{2}}}\right)
$$

as a function of 'amplitude' $C_{1}$ and 'phase' $\Phi$. 
Equations (22), (23) are used to transform the original system (16) into the form with rotating phases:

$$
\begin{array}{ll}
C_{1}^{\prime}=\Delta Q+\mathbf{O}\left(\mu^{2}\right), & \Phi^{\prime}=\omega-\omega \Delta F+\mathbf{O}\left(\mu^{2}\right) \\
\rho^{\prime}=-\frac{\Delta U \sin \theta}{n_{1}}+\mathbf{O}\left(\mu^{2}\right), & \theta^{\prime}=n_{1}-\frac{\Delta U \cos \theta}{\rho n_{1}}+\mathbf{O}\left(\mu^{2}\right), \quad \varphi^{\prime}=1
\end{array}
$$

where:

$$
\begin{aligned}
& Q=\frac{H}{n_{1}}\left(\sqrt{1-\frac{1}{C_{1}^{2}}} \cos \Xi+\frac{1}{C_{1} \sqrt{ } 1+\chi(\hat{\Phi}) p^{2}} \sin \Xi\right)=\mathbf{O}(1), \\
& F=\frac{H}{C_{1} n_{1}^{2}}\left\{\frac{\sin \Xi}{\sqrt{\left(1+\chi(\hat{\Phi}) p^{2}\right)\left(1-\frac{1}{C_{1}^{2}}\right)}}-\frac{\Delta p^{2}\left\{\Phi-2 \pi\left(\left[\frac{\Phi}{2 \pi}\right]+\chi(\hat{\Phi})\right)\right\}}{\pi C_{1}\left[1+p^{2}\left(1-\frac{1}{C_{1}^{2}}\right)\right]}\right. \\
& \left.\times\left[\left(1-\frac{1}{C_{1}^{2}}\right) \cos \Xi+\frac{1}{C_{1}} \frac{\sqrt{1-\frac{1}{C_{1}^{2}}}}{\sqrt{1+\chi(\hat{\Phi}) p^{2}}} \sin \Xi\right]\right\}=\mathbf{o}(1), \\
& H=b \sin (\varphi+v)-2 \frac{\eta}{\Delta} C_{1} n_{1}\left[\frac{1}{C_{1} \sqrt{ } 1+\chi(\hat{\Phi}) p^{2}} \sin \Xi+\sqrt{1}-\frac{1}{C_{1}^{2}} \cos \Xi\right] \\
& -\frac{\rho^{2} n_{1}^{2}}{4}(1-3 \cos 2 \theta) \text {, }
\end{aligned}
$$

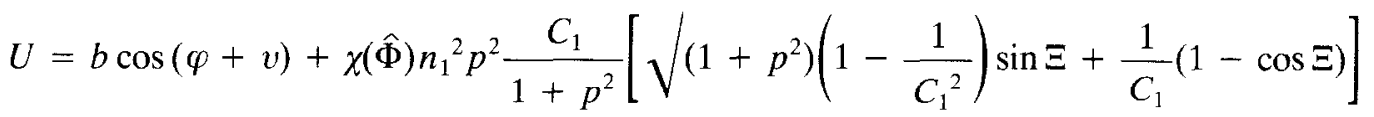

$$
\begin{aligned}
& \times\left(\rho \cos \theta+\frac{f}{\Delta}\right)+2 \frac{\eta}{\Delta} \rho n_{1} \sin \theta, \\
& b=\frac{1}{\Delta^{2}} \sqrt{ } a^{2}+2 a s_{\mathrm{T}}\left(n_{1}^{2}-\kappa\right) \cos \beta+s_{T}^{2}\left(n_{1}^{2}-\kappa\right)^{2}, \\
& v=\arccos \left(\frac{a+s_{T}\left(n_{1}{ }^{2}=\kappa\right) \cos \beta}{\sqrt{a^{2}+2 a s_{T}\left(n_{1}{ }^{2}-\kappa\right) \cos \beta+s_{T}{ }^{2}\left(n_{1}{ }^{2}-\kappa\right)^{2}}}\right) .
\end{aligned}
$$

Note that the variables $\rho$ and $C_{1}$ are slow, which conforms with their meaning of amplitudes. Equations (24) for variables $\theta, \Phi$ differ from those for rotations with a constant speed by small terms, and all right-side terms are $2 \pi$-periodic functions of them; therefore, $\theta, \Phi$ have the meaning of phases. The important difference betwcen the phases $\theta$ and $\Phi$ is that $\theta$ has a constant frequency $n_{1}$ (see equation (17)), which means that the rotor horizontal motion is quasi-linear, while $\Phi$ has a frequency $\omega=\omega\left(C_{1}\right)$, dependent on the 'amplitude' $C_{1}$. Such a disparity is due to the assumed vertical-down direction of the radial (side-load) force $P$.

The systems of the type (24) are known in the litcraturc [21] as the systems with multi-dimensional rotating phases, and according to the corresponding theorem, allow the application of the Averaging Method. The latter produces a variable transformation close to identical, which converts the original equations (24) with accuracy up to the small terms of the assumed power of the small parameter into a system with its right-side terms 
dependent only on slow variables. The first approximation of the new system right-side terms is the result of simple averaging of the corresponding right-side terms of cquation (24) over the independent rotating phases.

\section{RESONANCES IN THE SYSTEM WITH THREE ROTATING PHASES}

The Fourier analysis of the right-side terms of (24) shows that they include the following combinations of the phases $\Phi, \theta, \varphi$ :

$$
\varphi . \quad \Phi, \theta, \varphi \pm k \Phi, \theta \pm k \Phi, 2 \theta \pm k \Phi, \varphi \pm \theta \quad(k=1,2,3, \ldots) .
$$

This means that possible resonances occur when:

$$
1 \pm k \omega=0, \quad n_{1} \pm k \omega=0, \quad 2 n_{1} \pm k \omega=0, \quad 1 \pm n_{1}=0 \quad(k=1,2,3, \ldots) .
$$

As it results from the expression (23), the ratio $\omega / n_{1}$ is contained within the following limits:

$$
1 \leqslant \frac{\omega}{n_{1}} \leqslant \sqrt{1}+p^{2}
$$

Taking the inequality (28) into account, the number of possible resonances (27) is reduced to the following:

$$
1 \pm n_{1}=0, \quad 2 n_{1} \pm \omega=0, \quad 1 \pm k \omega=0 \quad(k=1,2,3, \ldots)
$$

and their linear combinations. In order to find stationary resonance regimes, the neighborhood of each resonance will be investigated by the averaging method. The steady state can only be achieved under the conditions of balance between the supplied and dissipated energies. From this standpoint especially important are the equations (24) for $C_{1}^{\prime}$ and $\rho^{\prime}$, since these variables, in the very real sense, are the amplitudes of the corresponding vertical and horizontal vibrations (in a general case, amplitudes of vibration in the direction of the radial (side-load) force and in the orthogonal direction). A simple analysis shows that all right-side terms of these equations can be split into three groups:

(1) terms responsible for the energy delivery from the external source;

(2) terms responsible for the energy dissipation;

(3) terms responsible for the energy exchange between vertical and horizontal modes.

It is important to note that if, after averaging, cquation (24) for $C_{1}^{\prime}$ includes as a right-side term only a dissipative term, the corresponding stationary regime does not exist, because the system does not allow for a trivial solution $C_{1}=0$. On the other hand, the corresponding equation for $\rho^{\prime}$ allows the zero solution; therefore, the stationary resonance regime is possible if there is only a dissipation term in the equation for $\rho^{\prime}$.

The above consideration can be summarized in the form of the following conditions for the stationary resonance solution.

- If there exists a source of energy in one of the equations (24), the resulting regimes are: $1 \pm n_{1}=0$ and $/$ or $1 \pm k \omega=0(k=1,2,3, \ldots)$.

- If there exists at least one more term besides the dissipative term in the equation (24) for $C_{1}^{\prime}$, the resulting regimes are: $1 \pm k \omega=0(k=1,2,3, \ldots)$ and/or $2 n_{1} \pm \omega=0$.

Taking into account the inequality (28), this means that the system possibly allows the stationary resonant solutions for the case of $1 \pm k \omega=0(k=1,2,3, \ldots)$, or for the case of a combinational resonance: $1 \pm n_{1}=0$ and $2 n_{\mathrm{L}} \pm \omega=0$. 
The first resonance occurs when the rotative speed of the shaft $\Omega$ is close to the rotor first natural frequency $v_{1}$, and the vertical response frequency $\omega \Omega$ is close to $2 v_{1}$ (horizontal mode). This closeness can be described by the following relations:

$$
\begin{aligned}
& n_{1}-1=\xi=\mathbf{O}(\mu) \\
& C_{1}-C_{a}=\mathbf{O}(\sqrt{ } \mu)
\end{aligned}
$$

where $\xi$ is a frequency detuning, and $C_{\mathrm{a}}$ is a solution of the following equation:

$$
\omega\left(C_{a}\right)-2=0 .
$$

Equations (24) are averaged over the sole independent rotating phase $\varphi$ in the described proximity of the vertical resonance, and then stable stationary solutions of the averaged equations are found (for averaging technique and stability criteria, see for example ref. [26]):

In the range $\sqrt{3}<p<\sqrt{6}$ :

$$
\begin{aligned}
\phi \approx \pi-\arcsin \left(\frac{4 \frac{\eta}{\Delta} G\left[\frac{\xi}{\Delta}+\frac{C_{a}}{2}\left(G-C_{a}\right)+B\right]^{2}}{b^{2} A}\right)-2 \arccos \frac{1}{C_{a}}+2 v \\
+2 \arctan \left(\frac{\left.\frac{\eta}{\Delta}\left\{1-4 G \frac{B}{A b^{2}}\left[\frac{\xi}{\Delta}+\frac{C_{a}}{2}\left(G-C_{a}\right)+B\right]^{2}\right\}\right)}{\left[\frac{\xi}{\Delta}+\frac{C_{a}}{2}\left(G-C_{a}\right)+B\right]}\right) \\
\quad \tan (\gamma-v)=-\frac{\frac{\eta}{\Delta}\left\{1-4 G \frac{B}{A b^{2}}\left[\frac{\xi}{\Delta}+\frac{C_{a}}{2}\left(G-C_{a}\right)+B\right]^{2}\right\}}{\left[\frac{\xi}{\Delta}+\frac{C_{a}}{2}\left(G-C_{a}\right)+B\right]} \\
\rho^{2}=\frac{b^{2}+8 \frac{\eta^{2}}{\Delta^{2}} G \frac{B}{A}\left\{1+\frac{2 G B}{b^{2} A}\left[\frac{\xi}{\Delta}+\frac{C_{a}}{2}\left(G-C_{a}\right)\right]\left[\frac{\xi}{\Delta}+\frac{C_{a}}{2}\left(G-C_{a}\right)+B\right]^{2}\right\}}{\left.4\left[\frac{\xi}{\Delta}+\frac{C_{a}}{2}\left(G-C_{a}\right)+B\right]^{2}+\frac{\eta^{2}}{\Delta^{2}}\right]}
\end{aligned}
$$

In the range $p>\sqrt{6}$ :

$$
\begin{aligned}
\phi \approx \arcsin & \left(\frac{4 \frac{\eta}{\Delta} G\left[\frac{\xi}{\Delta}+\frac{C_{a}}{2}\left(G-C_{a}\right)-B\right]^{2}}{b^{2} A}\right)-2 \arccos \frac{1}{C_{a}}+2 v \\
& +2 \arctan \left(\frac{\frac{\eta}{\Delta}\left\{1-4 G \frac{B}{A b^{2}}\left[\frac{\xi}{\Delta}+\frac{C_{a}}{2}\left(G-C_{a}\right)-B\right]^{2}\right\}}{\left[\frac{\xi}{\Delta}+\frac{C_{a}}{2}\left(G-C_{a}\right)-B\right]}\right),
\end{aligned}
$$




$$
\begin{gathered}
\tan (\gamma-v)=-\frac{\frac{\eta}{\Delta}\left\{1-4 G \frac{B}{A b^{2}}\left[\frac{\xi}{\Delta}+\frac{C_{a}}{2}\left(G-C_{a}\right)-B\right]^{2}\right\}}{\left[\frac{\xi}{\Delta}+\frac{C_{a}}{2}\left(G-C_{a}\right)-B\right]}, \\
\rho^{2}=\frac{b^{2}+8 \frac{\eta^{2}}{\Delta^{2}} G \frac{B}{A}\left\{1-\frac{2 G B}{b^{2} A}\left[\frac{\xi}{\Delta}+\frac{C_{a}}{2}\left(G-C_{a}\right)\right]\left[\frac{\xi}{\Delta}+\frac{C_{a}}{2}\left(G-C_{a}\right)-B\right]^{2}\right\}}{\left.4\left[\frac{\xi}{\Delta}+\frac{C_{a}}{2}\left(G-C_{a}\right)-B\right]^{2}+\frac{\eta^{2}}{\Delta^{2}}\right]}, \\
\Phi=2 \varphi+\phi ; \quad \theta=\varphi+\gamma,
\end{gathered}
$$

where:

$$
\begin{aligned}
& A\left(C_{a}, p^{2}\right)=\frac{3\left(1-\frac{1}{C_{a}^{2}}\right)^{3 / 2}\left(6-p^{2}\right)}{\pi\left(p^{2}-3\right)}, \\
& B\left(C_{a}, p^{2}\right)=\frac{C_{a} p^{2} \sqrt{1-\frac{1}{C_{a}^{2}}}\left[1+p^{2}-\frac{1}{C_{a}^{2}}\left(3 p^{2}-5\right)\right],}{\pi\left(1+p^{2}\right)\left(p^{2}-3\right)} \\
& G\left(C_{a}, p^{2}\right)=\left\{C_{a}+\frac{2 p^{2}}{\pi\left(1+p^{2}\right)}\left[\frac{1}{C_{a}} \arccos \left(\sqrt{1-\frac{1}{C_{a}^{2}}}\right)+\sqrt{1-\frac{1}{C_{a}^{2}}}\right]\right\} .
\end{aligned}
$$

A numerical example of $\rho$ calculated by using equations (32) is given in Fig. 6. The relative magnitudes of the horizontal and vertical amplitudes are presented in Fig. 7. An example of vertical and horizontal phase behavior based on (32) is presented in Fig. 8.

The vertical resonance, $\omega=1$ occurs at higher rotative speed then the horizontal one.

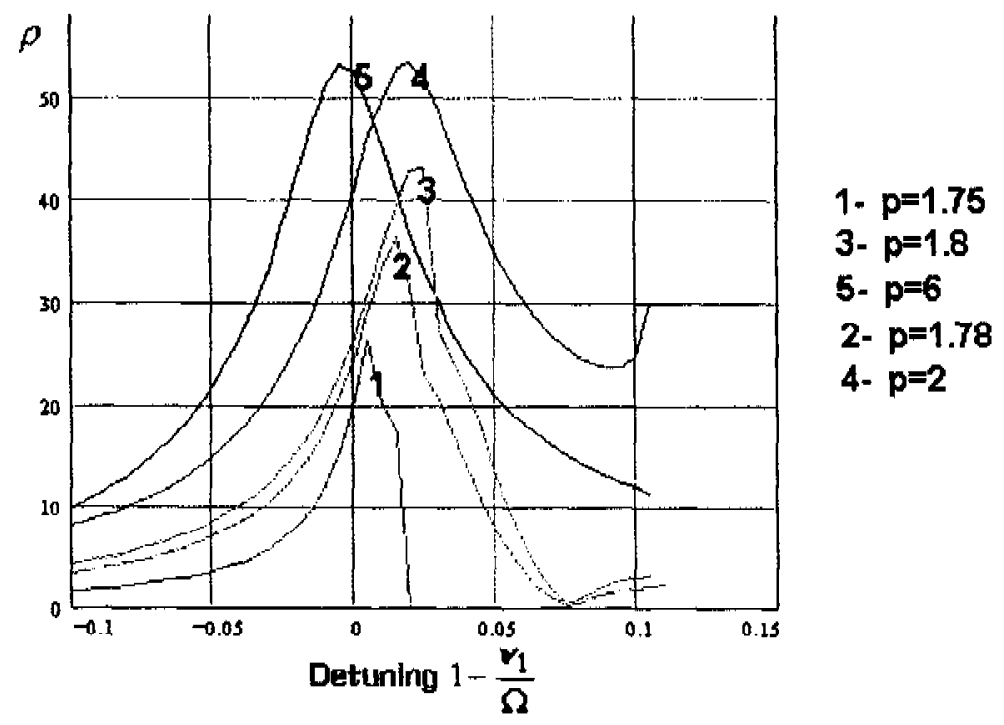

Fig. 6. The rotor overall horizontal amplitude-to-nondimensional clearance ratio versus detuning for the horizontal mode, calculated for the following set of parameters: $\Lambda=0.2, \eta=0.03, b=16$ and variable $p$. 


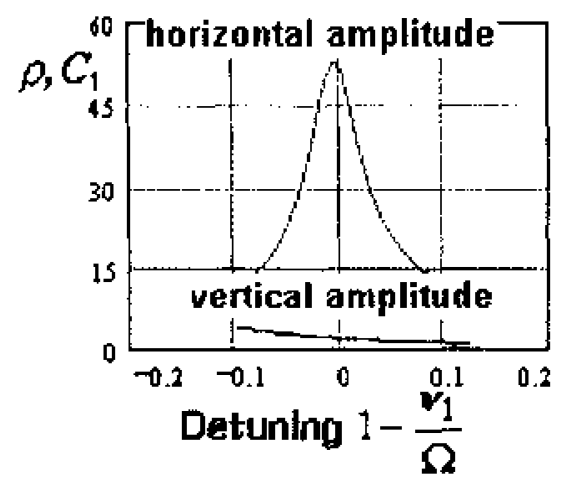

Fig. 7. The overall horizontal and vertical amplitudes-to-nondimensional cicarance ratios versus detuning for the horizontal mode, calculated for the following set of parameters: $\Delta=0.2, p=4, \eta=0.03, b=$ If.
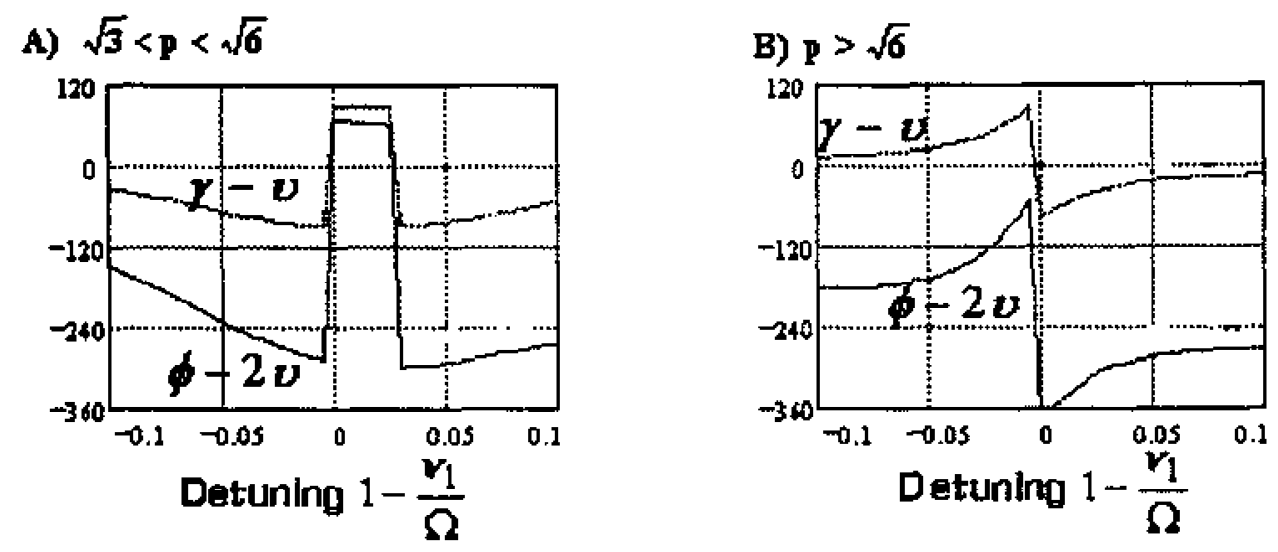

Fig. 8. The horizontal $\gamma-v$ and vertical $\phi-2 v$ phases of the row response versus detuning for the horizontal mode, calculated using the following sets of parameters: $\Delta=0.2, \eta=0.03, b=16$ and $p=2$ for case (A), $n \rightarrow 4$ for case (B).

The arca around this resonance is referred to as a main vertical mode. The vertical resonances $1 \pm k \omega=0(k=2,3, \ldots)$, together with their vicinities, are referred to as fractional vertical modes. In general all arcas around the resonances can be described by the second equation (30), where $C_{a}$ is a root of the following equation (Fig. 9):

$$
1-k \omega=0 \quad(k=1,2,3, \ldots) .
$$

Equations (24) after the averaging in proximities of vertical resonances (main and fractional) allow for the following stable stationary solutions:

and for $G>0(33)$ :

$$
\rho=0 \text {. }
$$

$$
\begin{gathered}
\Phi=\frac{\varphi}{k}-2 \pi \frac{i}{k}+\frac{\Theta+v}{k}-\frac{1}{k} \arccos \frac{\pi k\left(1-n_{1}^{2}\right)\left[\left(1+p^{2}\right) n_{\mathrm{I}}^{2}-1\right] \frac{\eta}{\Delta} G}{b n_{1} p^{2}\left(\sqrt{\left.1-\frac{1}{C_{a}^{2}} \cos \Theta+\frac{n_{1}}{C_{a}} \sin \Theta\right)}\right.} \\
\quad\left(i=0,1, \ldots, \quad C_{1}=C_{a}\right.
\end{gathered}
$$




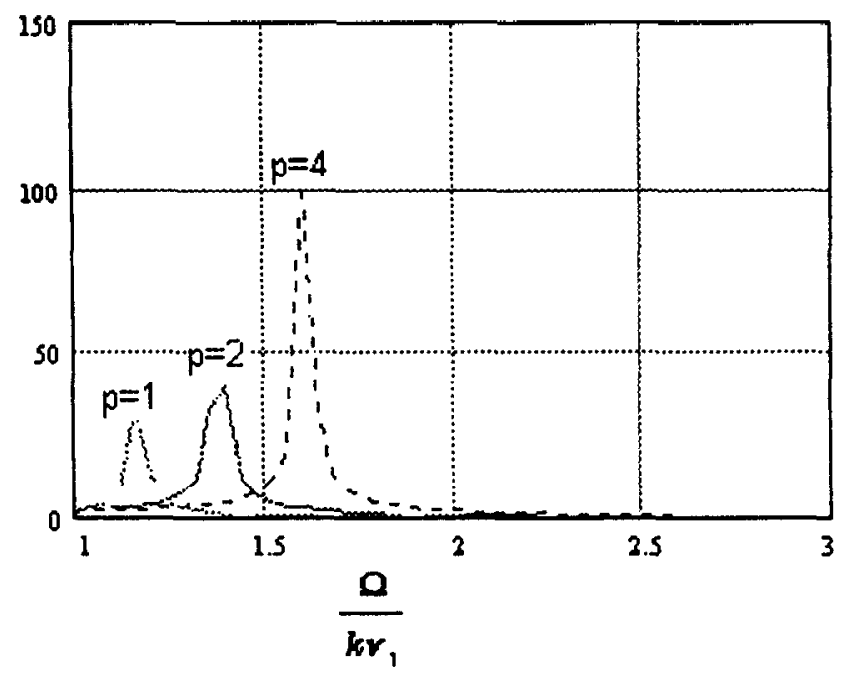

Fig. 9. The overall vertical amplitude-to-nondimensional clearance ratio versus rotative speed to $k$ times natural frequency $v_{1}$ ratio for different values of stiffening coefficient $p$ for the vertical modes. Other parameters do not affect vertical amplitudes.

while for $G<0$ :

$$
\begin{gathered}
\Phi=\frac{\varphi}{k}-2 \pi \frac{i}{k}+\frac{\Theta+v}{k}+\frac{1}{k} \arccos \frac{\pi k\left(1-n_{1}^{2}\right)\left[\left(1+p^{2}\right) n_{1}^{2}-1\right] \frac{\eta}{\Delta} G}{b n_{1} p^{2}\left(\sqrt{\left.1-\frac{1}{C_{a}^{2}} \cos \Theta+\frac{n_{1}}{C_{a}} \sin \Theta\right)}\right.} ; \\
(i=0,1, \ldots, k-1) \\
\rho=0, \quad \theta=\left[n_{1}+\Delta \frac{C_{a}}{2}\left(G-C_{a}\right)\right] \tau+\gamma, \quad \varphi=\tau+\delta, \quad C_{1}=C_{a}
\end{gathered}
$$

where:

$$
\Theta=\frac{1}{n_{1}}\left(\pi-\arccos \frac{1}{C_{a}}\right) .
$$

The boundary between positive and negative values of the function $G$ (Eqns (33)) in the plane of parameters $p, \Omega / k v_{1}$ is shown in Fig. 10 .

The relationship of the vertical response phase $\Phi-(\varphi+v) / k$ versus rotative speed ratio for the vertical modes $k=1$ and $k=2$, calculated for a particular set of parameters $\Delta, p$, $\eta$ and $b$, is presented in Fig. 11.

As it results from the resonance relation (34), Fig. 9 and equation (35) the vertical modes are displayed as a sequence of rotor vertical overall vibration amplitude peaks occurring at the rotative speed-to-natural-frequency $v_{1}$ ratios close to integer numbers $k=1,2,3, \ldots$ Amplitudes of the horizontal responses are much smaller than those of vertical responses. The periods of oscillations in each case is equal to $k$ times rotations, which corresponds to fractional $1 / k \times$ regimes. The number of possible stable regimes starts from one and increases with $k$. These regimes differ from each other by the constant phase $\Phi$ shift $2 \pi i / k(i=0,1,2, \ldots, k-1)$. 


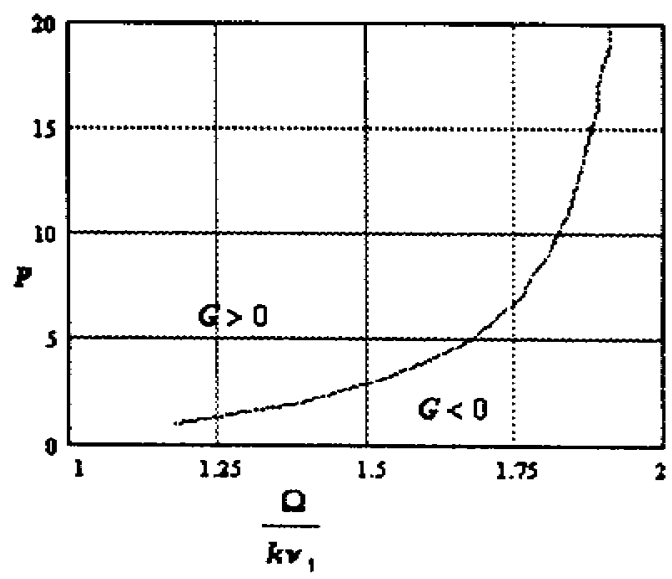

Fig. 10. The boundary between positive and negative values of $G$ (equations (33)) as a function of the parameters $\rho$ and $\Omega / k v_{1}$.

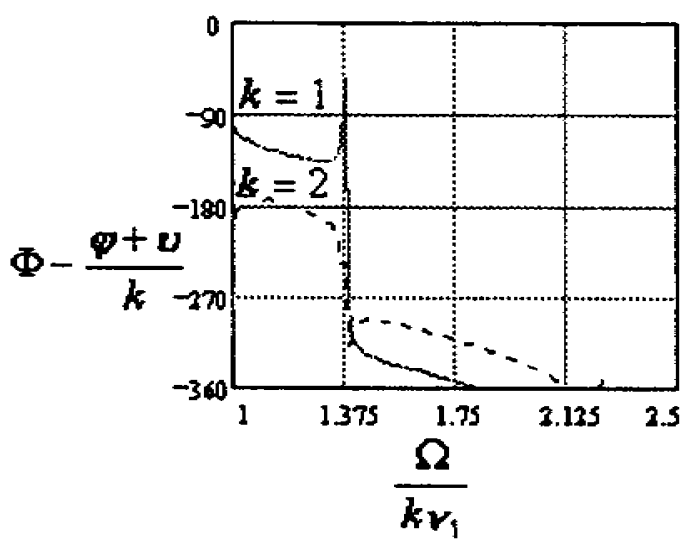

Fig. 11. Vertical phase $\Phi-(\varphi-v) / k$ of the rotor response versus rotative speed to $k$ times natural frequency $v_{\mathrm{j}}$ ratio for vertical modes $k=1$ and $k=2$, calculated for the following set of parameters: $\Delta=0.2, p=2, \eta=0.03$, $b=16$.

\section{THERMAL BOW}

After solving the mechanical problem in the vicinities of the horizontal and vertical resonances, the expressions for the heat rate $\hat{g}$ can be obtained. This allows for approaching the problem of thermal bow behavior in the slow time scale. In order to accomplish it, the first equation (19) is multiplied by $\hat{r}^{2}$ and integrated over $\hat{r}$ from 0 to 1 . After some transformations, this leads to the following expression:

$$
\frac{\mathrm{d} \widetilde{T}}{\mathrm{~d} \tau}=\varepsilon\left[\frac{R \hat{g}}{\hat{k}}-\left.\left(\frac{\hat{h} R}{\hat{k}}+1\right) \hat{T}\right|_{\hat{f}=1}\right] .
$$

Equation (37) has a standard form for which the averaging method [21] can be applied. At the first approximation, the application of this method means averaging of the right-side term of (37) over independent rotating phases:

$$
\frac{\mathrm{d} \widetilde{T}}{\mathrm{~d} \tau}=\varepsilon\left[\frac{R(\hat{g})}{\hat{k}}-\left.\left(\frac{R \hat{h}}{\hat{k}}+1\right)\langle\hat{T}\rangle\right|_{\hat{r}=1}\right]+\ldots
$$


where $\langle\ldots\rangle$ is a notation for the average value of the variable over the mechanical oscillation period.

Based on (19), the following relationship can be established:

$$
|\langle\hat{T}\rangle-4 \widetilde{T}|=\mathbf{O}\left(\frac{\mathrm{d}\langle\hat{g}\rangle}{\mathrm{d} \tau}\right)=\mathbf{O}(\mu \widetilde{T}) .
$$

Using (39), (38) can be rewritten in the following form:

$$
\frac{\mathrm{d} \widetilde{T}}{\mathrm{~d} \tau}=\varepsilon\left[\frac{R\langle\hat{g}\rangle}{\hat{k}}-4\left(\frac{R \hat{h}}{\hat{k}}+1\right) \widetilde{T}\right]+\ldots
$$

Using (12), (40) can be presented as follows:

$$
\frac{\mathrm{d} \hat{\rho}_{T}}{\mathrm{~d} t}+\frac{\hat{\rho}_{T}}{D_{T}}=\frac{\chi_{T} L}{\hat{h} R+\hat{k}}\left(\frac{l+l_{2}-\left|l-l_{2}\right|}{2 L}-\frac{l l_{2}}{L^{2}}\right) \frac{\langle\hat{g}\rangle}{D_{T}}+\ldots
$$

where the constant

$$
D_{T}=\frac{R^{2}}{4 \hat{K}\left(\frac{R \hat{h}}{\hat{k}}+1\right)}
$$

has a physical meaning of time constant of the thermal system. Equation (41) has almost the same structure as the equation in ref. [6], but differs from it by its vectorial (in a sense of complex numbers) character and distributed thermal bow of the shaft. As follows from (19) and (22), $\hat{g}$ is a function of the 'fast' variables $\varphi$ and $\Phi$ and the 'slow' variable $C_{1}$. Since (41) for the thermal bow contains the function $\langle\hat{g}\rangle$, an averaging of $\hat{g}$ over phases $\varphi$ and $\Phi$ has to be performed. Using (22) and (32), it is easy to show that the expression (19) for $\hat{g}$, averaged over two rotations (two rotations constitute one oscillation in the case of the horizontal mode) gives zero value for the horizontal mode. It means that in this particular mode, the heat is generated symmetrically, and the thermal bow does not appear. For the vertical modes, using (19), (22), (35), the following expressions for $\langle\hat{g}\rangle$ are obtained after averaging:

$$
\begin{aligned}
\langle\hat{g}\rangle=f \Omega c\left(K_{2}+K_{3}\right) \Delta C_{a} n_{1} \frac{\sqrt{1-\frac{1}{C_{a}^{2}} \cos \Theta+\frac{n_{1}}{C_{a}} \sin \Theta}}{\pi k\left[\left(1+p^{2}\right) n_{1}^{2}-1\right]} \\
\quad \times \exp j\left[v+\frac{\pi}{2}-\operatorname{sign}(G) \arccos -\pi k\left(1-n_{1}^{2}\right)\left[\left(1+p^{2}\right) n_{1}^{2}-1\right] \frac{\eta}{\Delta} G\right] \\
b n_{1} p^{2}\left(\sqrt{\left.1-\frac{1}{C_{a}^{2}} \cos \Theta+\frac{n_{1}}{C_{a}} \sin \Theta\right)}\right]
\end{aligned}
$$

Taking (42) into account, (41) can be rewritten in terms of vector $\mathbf{b}=b \mathrm{e}^{j v}$ (see (25)):

$$
\frac{\mathrm{d} \mathbf{b}}{\mathrm{d} t}+\frac{\mathbf{b}}{D_{T}}=\frac{1}{D_{T}} \frac{a}{\Delta^{2}}+j \frac{\Lambda}{D_{T}}\left[\frac{\frac{\eta}{\Delta} G\left(1-n_{1}^{2}\right)}{n_{1} b p^{2}}-j \operatorname{sign}(G) \sqrt{F^{2}-\left[\frac{\frac{\eta}{\Delta} G\left(1-n_{1}^{2}\right)}{n_{1} b p^{2}}\right]}\right] \frac{\mathbf{b}}{b}
$$


where

$$
\hat{F}=\frac{\sqrt{1-\frac{1}{C_{a}^{2}} \cos \Theta+\frac{n_{1}}{C_{a}} \sin \Theta}}{\pi k\left[\left(1+p^{2}\right) n_{1}^{2}-1\right]}, \quad \Lambda=\frac{\chi_{T} l_{2}}{\hat{h} R+\hat{k}}\left(1-\frac{l_{2}}{L}\right) \frac{f}{\Delta} \Omega n_{1}\left(K_{2}+K_{3}\right) C_{a}\left(n_{1}^{2}-\kappa\right)
$$

or in terms of the vector length $b$ and phase $v$ :

$$
\begin{gathered}
\left.\dot{b}+\frac{b}{D_{T}}=\frac{1}{D_{T}}\left(\frac{a}{\Lambda^{2}} \cos v+\Lambda \operatorname{sign}(G) \sqrt{\hat{F}^{2}-\left[\frac{\frac{\eta}{\Delta} G\left(1-n_{1}^{2}\right)}{n_{1} b p^{2}}\right]}\right]^{2}\right), \\
b \dot{v}=\frac{1}{D_{T}}\left(-\frac{a}{\Delta^{2}} \sin v+\Lambda \frac{\frac{\eta}{\Delta} G\left(1-n_{1}^{2}\right)}{n_{1} p^{2} b}\right) .
\end{gathered}
$$

At the beginning of rub, the thermal bow does not exist:

$$
\left.b\right|_{t=0}=\frac{a}{\Delta^{2}},\left.\quad v\right|_{t=0}=0 .
$$

In order to describe the behavior of the thermal bow close to the starting point, it is logical to assume that $\left|b-a / \Delta^{2}\right| \ll 1,|v| \ll 1$. According to these assumptions, (45) can be linearized as follows:

$$
\begin{gathered}
\frac{\mathrm{d}\left(b-\frac{a}{\Delta^{2}}\right)}{\mathrm{d} t}+\frac{1}{D_{T}}\left[1-\operatorname{sign}(G) E_{1}\right]\left(b-\frac{a}{\Delta^{2}}\right) \approx \frac{\Lambda}{D_{T}} \operatorname{sign}(G) \sqrt{\hat{F}^{2}-\left[\frac{\frac{\eta}{\Delta} G\left(1-n_{1}^{2}\right) \Delta^{2}}{n_{1} a p^{2}}\right]} \\
\dot{v}+\frac{v}{D_{T}} \approx \frac{1}{D_{T}}\left[\Lambda \frac{\frac{\eta}{\Delta} G\left(1-n_{1}^{2}\right) \Delta^{4}}{n_{1} p^{2} a^{2}}-E_{2} \operatorname{sign}(G)\left(b-\frac{a}{\Delta^{2}}\right)\right]
\end{gathered}
$$

where:

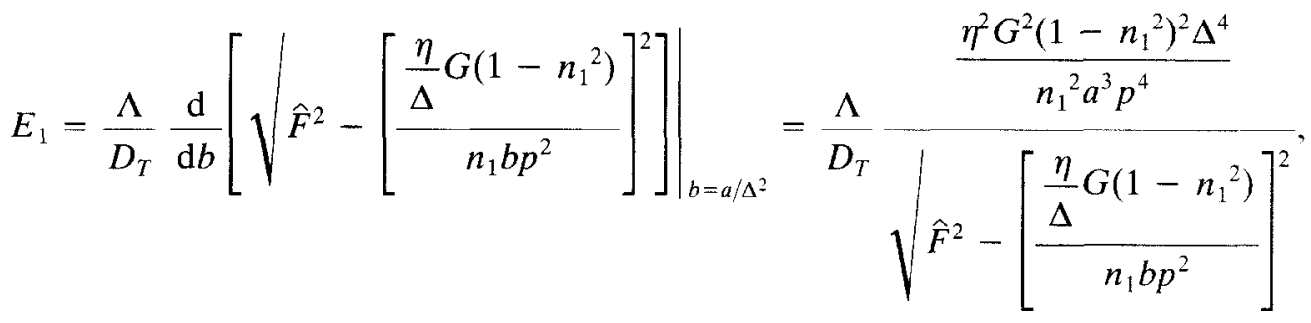

$$
\begin{aligned}
& E_{2}=-\left.\frac{\Lambda}{D_{T}} \frac{\mathrm{d}}{\mathrm{d} b}\left[\frac{\frac{\eta}{\Delta}|G|\left(1-n_{1}^{2}\right)}{n_{1} b p^{2}}\right]\right|_{b=a / \Delta^{2}}=2 \frac{\Lambda}{D_{T}} \frac{\eta|G|\left(1-n_{1}^{2}\right) \Delta^{5}}{n_{1} a^{3} p^{2}}>0 .
\end{aligned}
$$

Together with the initial conditions (46) equations (48) determine the following solution: 


$$
\begin{aligned}
b=\frac{a}{\Delta^{2}}+ & \frac{\Lambda \operatorname{sign}(G)}{1-E_{1} \operatorname{sign}(G)} \hat{F}^{2}-\left[\frac{\frac{\eta}{\Delta} G\left(1-n_{1}^{2}\right) \Delta^{2}}{n_{1} a p^{2}}\right]^{2}\left[1-\exp \left(-\left[1-E_{1} \operatorname{sign}(G)\right] \frac{t}{D_{T}}\right)\right] \\
v= & \Lambda \operatorname{sign}(G)\left[\frac{\Delta^{2}}{a} \frac{\frac{\eta}{\Delta}|G|\left(1-n_{1}^{2}\right) \Delta^{2}}{n_{1} p^{2} a}-\frac{E_{2} \operatorname{sign}(G)}{1-E_{1} \operatorname{sign}(G)}\right]\left[1-\exp \left(-\frac{t}{D_{T}}\right)\right] \\
& +\frac{\Lambda E_{2} \operatorname{sign}(G)}{E_{1}\left[1-E_{1} \operatorname{sign}(G)\right]}\left[\exp \left[-\left[1-E_{1} \operatorname{sign}(G)\right] \frac{t}{D_{T}}\right]-\exp \left(-\frac{t}{D_{T}}\right)\right]
\end{aligned}
$$

There are three possible cases of the thermal bow behavior, described by (49). They depend on the sign of the function $G$ and the function $1-E_{1} \operatorname{sign}(G)$ :

(1) $G>0,1-E_{1}>0$.

In this case, according to (49), the thermal bow is exponentially approaching the equilibrium value:

$\mathbf{b} \underset{i \rightarrow * x}{\longrightarrow} b \mathrm{e}_{s} \mathrm{e}^{i \psi_{\mathrm{x}}}$.

(2) $G>0,1-E_{1}<0$.

$$
\begin{aligned}
& b_{\infty}=\frac{a}{\Delta^{2}}+\frac{\Delta D_{T}}{1-E_{1} D_{T}} \sqrt{F^{2}-\left[\frac{\frac{\eta}{\Delta} G\left(1-n_{1}^{2}\right) \Delta^{2}}{n_{1} a p^{2}}\right]^{2}} \\
& v_{\infty}-\Lambda\left[\frac{\Delta^{2}}{a} \frac{\frac{\eta}{\Delta} \mid G\left(1-n_{1}^{2}\right) \Delta^{2}}{n_{1} p^{2} a}-\frac{E_{2}}{1-E_{1}}\right]
\end{aligned}
$$

In this case (49) describe unlimited exponential growth of the thermal bow amplitude with an exponential rotation of phase in the direction opposite to the direction of rotor rotation:

$$
\begin{aligned}
b= & \frac{a}{\Delta^{2}}+\frac{\Lambda}{E_{1}-1} \sqrt{\hat{F}^{2}-\left[\frac{\frac{\eta}{\Delta} G\left(1-n_{1}^{2}\right) \Delta^{2}}{n_{1} a p^{2}}\right]}\left[\exp \left[\left(E_{1}-1\right) \frac{t}{D_{T}}\right]-1\right] \\
v= & \Lambda\left[\frac{\Delta^{2}}{a} \frac{\frac{\eta}{\Delta}|G|\left(1-n_{1}^{2}\right) \Delta^{2}}{n_{1} p^{2} a}+\frac{E_{2}}{E_{1}-1}\right]\left[1-\exp \left(-\frac{t}{D_{T}}\right)\right] \\
& -\frac{\Lambda E_{2}}{E_{1}\left[E_{1}-1\right]}\left[\exp \left[\left(E_{1}-1\right) \frac{t}{D_{T}}\right]-\exp \left(-\frac{t}{D_{T}}\right)\right] .
\end{aligned}
$$

(3) $G<0,1-E_{\mathrm{I}} \operatorname{sign}(G)=1+E_{1}>0$.

In this case (49) describe exponentially decreasing amplitude of the thermal bow:

$$
b=\frac{a}{\Delta^{2}}-\frac{\Lambda}{1+E_{1}} \sqrt{F^{2}-\left[\frac{\frac{\eta}{\Delta} G\left(1-n_{\mathrm{l}}^{2}\right) \Delta^{2}}{n_{1} a p^{2}}\right]^{2}\left[1-\exp \left(-\left[1+E_{1}\right] \frac{t}{D_{I}}\right)\right]}
$$


The rotor vibration amplitude decreases until there is no more rotor-to-stator rubbing, and (49) is not valid any more. At this moment the initial pre-rub conditions may take over and lead to the rub, heating and thermal bowing again. The described scenario corresponds to the thermal bow oscillating regime.

All three cases are illustrated in Fig. 12. Note that for a fixed parameter $p$ the low values of $\Omega / k v_{1}$ (for example, if $p=2\left(\Omega / k v_{1}\right)<1.4$ ) correspond to one of two regimes: if $E_{1}<1\left(E_{1}\right.$ is a function of all parameters of the system as described by (36), (44) and (48), the thermal bow is exponentially approaching the equilibrium value (see (50)); if $E_{1}>1$, the thermal bow behaves as an unwinding spiral (see (51)). In the case of high values of the parameter $\Omega / k v_{1}$ (for $p=2\left(\Omega / k v_{1}\right)>1.4$ ), the only possible regime is the thermal bow oscillation.

\section{SUMMARY OF RESULTS}

This paper outlines the modeling of thermal/mechanical effects of one of the most destructive malfunctions in rotating machinery: the rotor-to-stator rub. The model of an isotropic rotor at its first lateral mode, with rotor-to-stator rub at a particular axial location is considered (see equation (1) and Fig. 1). It is assumed that, due to rub-related, localized heating and uneven temperature distribution, the thermal bow of the shaft results. This assumption correlates the thermal bow with the temperature distribution (see Fig. 4 and equation (12)). The temperature distribution due to the rub-related heating is given by the heat transfer equation with corresponding boundary conditions (see (6)-(8)), where the heat rate density per unit area is determined by the normal force and rotor rotationgenerated friction during the rotor-to-stator contact. The thermal/mechanical problem is partially uncoupled by the assumption that the thermal process is relatively slow. As a result, the thermal bow remains in the mechanical equations as a parameter which can be considered as a constant. In addition, the assumption is made that the radial (side-load) force keeps the centerline position of the rotor close to the location of contact with the stator. The rotor static position is perturbed by small forces generated by the thermal bow and the original unbalance. The coefficient of surface dry friction between the rotor and the stator is assumed small. In order to reduce the system equations of motion into a

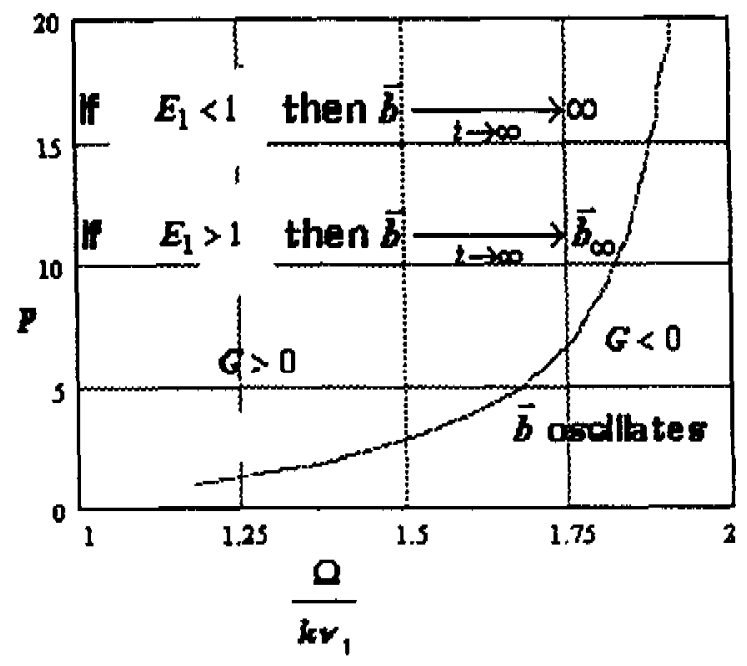

Fig. 12. Behavior of the combined unbalance vector $\mathbf{b}$ as a function of the thermal system function $E_{1}$, stiffness parameter $p$, and rotative speed ratio $\Omega / k v_{1}$. Note that $E_{1}$ depends on several system parameters (see (48)). 
standard form required for further analysis, new variables were introduced, one as the nondimensionalized distance from the rotor to stator at the axial location of the rub, and the other as an approximation to the nondimensionalized horizontal displacement (see (7)). The analysis of relative smallness of separate terms allows the presentation of the model in the form of equations with piecewise variable stiffness and 'small' right-side expressions (see (15)). These equations are then integrated using the generating approximation (neglecting right-side terms; see (20)). The obtained solution (see (22), (23) and Fig. 5) is used as a variable transformation which allows the presentation of the original system in the form with slow variables and rotating phases (see (24)). As it is shown in the Fig. 5, the nondimensionalized amplitude of the vertical response of the rotor can be expressed through the slow variable $C_{1}$ while $\rho$ represents nondimensionalized amplitude of the horizontal response. The rotating phase of the horizontal response $\theta$ has a constant frequency, equal to the rotor natural frequency $n_{1}$ when there is no contact with the stator. The rotating phase of the vertical response $\Phi$ has the frequency $\omega$ depending on the slow variable $C_{1}$ (see (23)). With assumed vertical direction of the radial force, the frequency of the vertical response is always higher than that of horizontal response. The Fourier analysis of small right-side terms of (24) and energy balance considerations allow for determination of possible resonances. The averaging technique applied to the system (24) in the proximity of these resonances, enhanced by the stability analysis, results in stable stationary solutions. These solutions describe analytically the rotor behavior in the proximity of the horizontal resonances (see (30)) which occur when $\Omega=-(1-1.05) v_{1}$ depending on the value of the stiffness parameter (see Fig. 6). They also describe the vicinity of the main vertical resonance (see (34) with $k=1$ ) which occurs when $\Omega=(1.3-1.8) v_{1}$ depending on the value of $p$, as well as the vicinities of the sequence of fractional vertical resonances (see (34) with $k=2,3, \ldots$ ) which occur when $\Omega=k(1.3-1.8) v_{1}$ (see Fig. 9). According to (15). (22), (23), the original variables, rotor horizontal and vertical displacements $x_{1}, y_{1}$ at the disk location, can be presented as follows:

$$
\begin{aligned}
& x_{\mathrm{i}} \approx\left(1+\frac{K_{3}}{K_{2}}\right)\left\{\left[c-\frac{P}{K_{1}\left(1+\frac{K_{3}}{K_{2}}\right)+K_{3}}\right] \rho \cos \theta\right. \\
& \left.-\rho_{T 2} \cos (\varphi+\beta)\left[1-\frac{\rho_{T 1}}{\rho_{T 2}\left(1+\frac{K_{3}}{K_{2}}\right)}\right]\right\} \text {, } \\
& y_{1} \approx\left(1+\frac{K_{3}}{K_{2}}\right)\left[c-\frac{P}{K_{1}\left(1+\frac{K_{3}}{K_{2}}\right)+K_{3}}\right]
\end{aligned}
$$

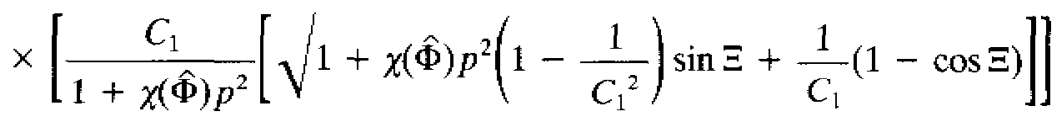

$$
\begin{aligned}
& -\rho_{T 2}\left(1+\frac{K_{3}}{K_{2}}\right) \sin (\varphi+\beta)\left[1-\frac{\rho_{T 1}}{\rho_{T 2}\left(1+\frac{K_{3}}{K_{2}}\right)}\right] \text {, } \\
& \Xi=\frac{n_{1} \sqrt{ } 1+\chi(\hat{\Phi}) p^{2}}{\omega}\left\{\Phi-2 \pi\left(\left[\frac{\Phi}{2 \pi}\right]+\chi(\hat{\Phi})\right)\right\}
\end{aligned}
$$


where for the case of the horizontal mode $\theta=\varphi+\gamma, \Phi=2 \varphi+\phi ; \rho, C_{1}, \gamma, \phi$ are given by (32). For the sequence $(k=1,2, \ldots)$ of vertical modes there is $\rho=0, \Phi=(\varphi+\phi) / k ; C_{1}$, $\phi$ are determined by (35). A sequence of typical orbits of the rotor centerline for the horizontal mode, and two vertical modes $(k=1)$ and $(k=2)$, calculated using (53) is shown in Fig. 13.

The developed analytical technique describes the system behavior in the narrow frequency zones in the vicinity of the resonances. As it follows from the obtained results, the motion in these zones has periodical (or quasi-periodical) character. In the remaining frequency range chaotic motion may occur. The chaotic motion is usually preceded by more complex periodic regimes which can be obtained by the same technique from the higher approximations consideration. It can be modeled numerically (see [23]). Note that the results regarding the mechanical behavior of the rubbing rotor are independent from the thermal problem, and represent a new approach to analytical modeling of such systems.

An application of the averaging technique allows derivation of the thermal bow equation (see (43)). It includes an average heat generation ratio. The latter was calculated based on the results obtained from the mechanical problem solution, as the friction force power per area unit averaged over the rotor largest vibration period of the rotor. Depending on the system parameters, the resulting vectorial relationship for the thermal bow exhibits one of three types of behavior.

- Asymptotic approach to the equilibrium state of the thermal bow;

- Increasing spiraling motion of the thermal bow in the direction opposite to rotation;

- Slow oscillations of the thermal bow.
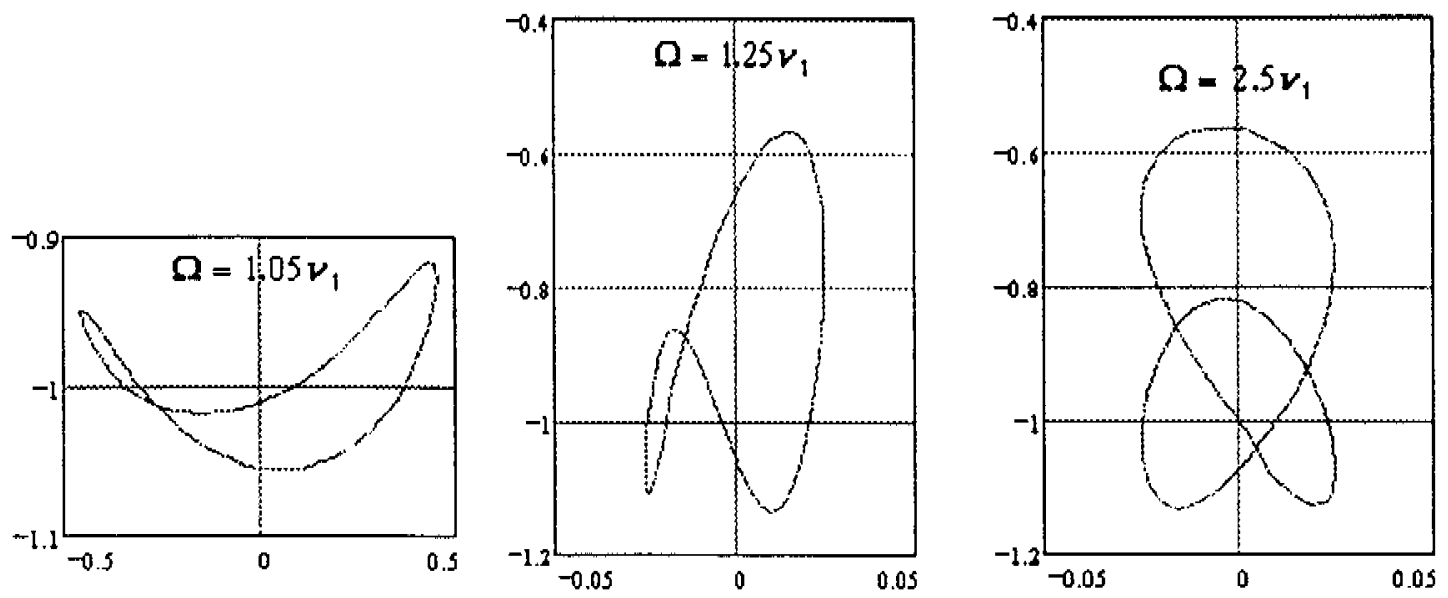

Fig. 13. A sequence of typical steady-state overall vibrational orbits of the rotor disk calculated for the following set of parameters:

$$
\begin{gathered}
P=1.1 c\left[K_{1}\left(1+\frac{K_{3}}{K_{2}}\right)+K_{3}\right], \quad \frac{D_{s}}{2 M \Omega}=0.03, \quad \frac{K_{2}}{K_{1}\left(1+\frac{K_{3}}{K_{2}}\right)+K_{2}}=2, \quad c M\left(1+\frac{K_{3}}{K_{2}}\right) \\
\rho_{T 2}=0.05 c, \quad \beta=30^{\circ}, \quad \frac{\rho_{I 1}}{\rho_{T 2}}=0.5\left(1+\frac{K_{3}}{K_{2}}\right)
\end{gathered}
$$

in a nondimensional form:

$$
\frac{x_{1}}{c\left(1+\frac{K_{3}}{K_{2}}\right)}, \quad \frac{y_{1}}{c\left(1+\frac{K_{3}}{K_{2}}\right)} .
$$


The results of the thermal bow behavior stand in qualitative agreement with results presented in refs [4] and [6]. They differ from them, however, in the conditions of transitions from one regime to the other (see (50)-(52) and Fig. 12). This difference is due to the more accurate description of the system's dynamics during rubbing that is presented in this paper.

\section{REFERENCES}

1. H. D. Taylor, Rubbing shafts above and below resonant speed, GE Technical Information Series, No. 16709 (1924).

2. B. L. Newkirk, Shaft rubbing, Mech. Eng. 48, 830 (1926).

3. R. P. Kroon and W. A. Williams, Spiral vibration of rotating machinery, Proc. 5th Int. Congr. Appl. Mech. John Wiley, New York, 712 (1939).

4. A. D. Dimarogonas, Newkirk effect: thermally induced dynamic instability of high speed rotors, ASME Paper 73-GT-26, Gas Turbine Conf., Washington D.C. (1973).

5. H. F. Black, Interaction on a whirling rotor with a vibrating stator across a clearance annulus, J. Mech. Eng. Sci. 10(1) (1968).

6. W. Kellenberger, Spiral vibrations due to the seal rings in turbogenerators. Thermally Induced Interaction Between Rotor and Stator, ASME Paper 79-DET-61, Design Engng Tech. Conf., St. Louis (1979).

7. N. S. Natho and O. E. Crenwelge, Case history of a steam turbine rotordynamic problem: theoretical versus experimental results, Vibration Institute Proceedings, Machinery Vibration Monitoring \& Analysis, pp. 81-89 (April 1983).

8. A. D. Dimarogonas, A study of the Newkirk effect in turbomachinery, WEAR, 28, 369-382 (1974).

9. Y. Hashemi, Vibration problems with thermally induced distortions in turbine-generators rotors, vibrations in rotating machinery, Third Int. Conf. Proceedings, IMechE, C271/84, York, UK (1984).

10. A. J. Smalley, The Dynamic Response of Rotors to Rubs During Startup, Rotating Machinery Dynamics, Vol. 2, Ed. by A. Muszynska and J. C. Simonis (1987).

11. A. Muszynska, Thermal/mechanical effect of rotor-to-stator rubs in rotating machinery, vibration of rotating systems, DE-vol. 60, ASME Design Technical Conf., Albuquerque, New Mexico (1993).

12. W. J. Sweets, Analysis of rotor rubbing, GE Technical Information Series, DF-66-LS-70 (1966).

13. A. D. Dimarogonas and S. A. Paipetis, Analytical Methods in Rotor Dynamics. Applied Science Publishers, London and New York (1983).

14. J. N. Goodier, Formulas for overall thermoelastic deformation, Proc. 3rd Int. Congr. Appl. Mech. John Wiley, New York, p. 343 (1958).

15. D. E. Bently, Forced subrotative speed dynamic action of rotating machinery, ASME Paper, 74-DET-16, Petroleum Mechanical Engineering Conference, Dallas, TX (1974).

16. D. W. Childs, Rub-induced parametric excitation in rotors, ASME Journal of Mechanical Design, ASME Paper 79-WA, DE-14, ASME Trans., v, 101, 649-644 (1979).

17. I. W. Childs, Fractional frequency rotor motion due to nonsymmetric clearance effects, Journal of Engineering for Power, 533-541 (1982).

18. Y. S. Choi and S. T. Noah, Nonlinear steady-state response of a rotor-support system, Journal of Vibration, Acoustics, Stress and Reliability in Design, 255-261 (1987).

19. A. Muszynska, W. D. Franklin and R. D. Hayashida, Rotor-to-stator partial rubbing and its effects on rotor dynamic response, The Sixth Workshop on Rotordynamic Instability Problem in High Performance Turbomachinery, NASA CP 3122, College Station, TX, pp. 345-362 (1990).

20. A. Muszynska, Rotor-to-stationary element rub-related vibration phenomena in rotating machinery, Literature Survey, The Shock and Vibration Digest, 21(3), 3-11 (1989).

21. N. N. Bogolubov and U. A. Mitropolsky, Asymptotic Methods in the Theory of Nonlinear Oscillations (in Russian). Nauka, Moskva (1974).

22. B. F. Zhuravlev, The equations of motion for the systems with ideal one sided restrictions (in Russian), $P M M$ (Applied Mathematics and Mechanics), 42(5), 781-788 (1978).

23. A. Petchenev and A. Fiddling, Hierarchy of the resonant motions of vibroimpacting system excited by the inertia source with limited power (in Russian), Mechanika Tverdogo Tela, 4 (1992).

24. P. Goldman and A. Muszynska, Dynamic effects in mechanical structures with gaps and impacting: order and chaos, Transactions of the ASME, Journal of Vibration and Acoustics, (to appear).

25. A. Muszynska, Shaft crack detection, Proc. of the 7th Machinery Dynamics Seminar, National Research Council, Edmonton, Alberta, Canada (1982).

26. P. Goldman and A. Muszynska, Resonances in the system of the interacted sources of vibration. Fornulation of problem and general results, Inter. J. Nonlinear Mech. 29(1), 49-63 (1994). 